CORRIGENDUM

doi:10.1038/nature 13550

\title{
Corrigendum: Mitofusin 2 tethers endoplasmic reticulum to mitochondria
}

Olga Martins de Brito \& Luca Scorrano

Nature 456, 605-610 (2008); doi:10.1038/nature07534

In Fig. 1a of this Article, the representative image of a volume-rendered three-dimensional reconstruction of a $\mathrm{z}$-stack of confocal images of endoplasmic-reticulum-targeted yellow fluorescent protein (ER-YFP) in a $M f n 2^{-/-}$cell expressing MFN2 $2^{\text {IYFFT }}$ and that of a $M f n 1^{-/-}$cell appear to be duplicated. Because the original raw data could not be located, we were unable to verify definitively whether the data in the original figure were indeed inadvertently duplicated. We therefore obtained new images under the same experimental conditions. The correct images of representative volume-rendered three-dimensional reconstruction of $\mathrm{z}$-stacks of confocal images of ER-YFP in $M f n 1^{-/-}$cells, $M f n 2^{-/-}$cells and $M f n 2^{-/-}$cells expressing MFN2 $2^{\text {IYFFT }}$ are shown in Fig. 1 of this Corrigendum. This does not affect any of our results.

Correspondence should be addressed to L.S. (luca.scorrano@unipd.it).
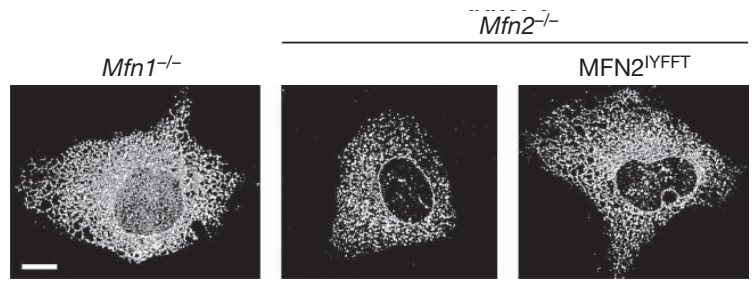

Figure $1 \mid$ This figure shows the results of the repeated experiments of Fig. 1a of this Article. Three-dimensional reconstructions of endoplasmic reticulum in mouse embryonic fibroblasts of the indicated genotype cotransfected with ER-YFP and the specified plasmids. Scale bar, $10 \mu \mathrm{m}$. 